\title{
Correction to: Risk factors associated with intensive care unit (ICU) admission and in-hospital death among adults hospitalized with COVID-19: a two-center retrospective observational study in tertiary care hospitals
}

\section{Azin Shayganfar ${ }^{1} \cdot$ Ramin Sami $^{2} \cdot$ Somayeh Sadeghi ${ }^{2} \cdot$ Mehrnegar Dehghan $^{3} \cdot$ Nilufar Khademi $^{3}$.} Reyhaneh Rikhtehgaran ${ }^{4} \cdot$ Reza Basiratnia $^{1} \cdot$ Felora Ferdosi $^{1} \cdot$ Somayeh Hajiahmadi ${ }^{1}$

Published online: 11 August 2021

(c) American Society of Emergency Radiology 2021

\section{Correction to: Emergency Radiology (2021) \\ https://doi.org/10.1007/s10140-021-01903-8}

The original article contains an error in Author name and her affiliation. The correct name is "Reyhaneh Rikhtehgaran" and her affiliation is "Department of Mathematical Sciences, Isfahan University of Technology, Isfahan, Iran”.

The original article has been corrected.

The original article can be found online at https://doi.org/10.1007/ s10140-021-01903-8.

Somayeh Hajiahmadi sohajiahmadi@gmail.com

1 Department of Radiology, School of Medicine, Isfahan University of Medical Sciences, Hezarjerib Avenue, Isfahan, Iran

2 Department of Internal Medicine, School of Medicine, Isfahan University of Medical Sciences, Isfahan, Iran

3 Isfahan University of Medical Sciences, Isfahan, Iran

4 Department of Mathematical Sciences, Isfahan University of Technology, Isfahan, Iran 